\title{
Algunos aspectos ecológicos y fitogeográficos de las especies de Cyperaceae en el Valle de México II. Análisis fitogeográfico ${ }^{1}$
}

\author{
SOCORRO GONZÁLEZ E. ${ }^{2,3}$ Y J. RZEDOWSKI ${ }^{2,4}$
}

RESUMEN. El análisis de las afinidades geográficas de los miembros de Cyperaceae pertenecientes a la flora del Valle de México revela que, en términos generales, las relaciones con el neotrópico prevalecen ampliamente sobre las que se pueden detectar con la región holártica, mientras que las especies de distribución restringida a México constituyen cerca de $30 \%$ del conjunto. Con países tan lejanos como Polonia y Liberia apenas existen especies en común, si bien a nivel de género la semejanza se mantiene por arriba de $50 \%$.

En escala más inmediata, la flora de ciperáceas del Valle muestra ligas evidentes con la de otras partes del Altiplano, pero tiene relativamente poco en común con la de las áreas de clima más cálido del país.

Los resultados apoyan la idea de Raven y Axelrod de que la familia evolucionó inicialmente en regiones tropicales y subtropicales y con posterioridad migró hacia el norte y el sur.

ABSTRACT. The analysis of the geographic affinities of the members of Cyperaceae belonging to the flora of the Valley of Mexico reveals that, in general terms, neotropical relationships prevail amply over the holarctic ones that were detected, while species with distribution restricted to Mexico amount to about $30 \%$ of the total number. With countries as distant as Poland and Liberia there are few species in common, although at the generic level, the similarity exceeds $50 \%$.

On a more regional scale, the cyperaceous flora of the Valley shows evident links with that of the Mexican High Plateau, but has relatively little in common with the flora of warmer parts of the country.

The results support the idea of Raven and Axelrod, who claim that the family evolved initially in tropical and subtropical areas and later migrated northward and southward.

\footnotetext{
1 Trabajo parcialmente subvencionado por el Consejo Nacional de Ciencia y Tecnología, en el marco del proyecto "Flora y Vegetación del Valle de México".

2 Laboratorio de Botánica Fanerogámica, Escuela Nacional de Ciencias Biológicas, Instituto Politécnico Nacional, 11410 México, D.F.

${ }^{3}$ Dirección actual: CiDIR.IPN Unidad Durango, Hidalgo 120, 34890 Vicente Guerrero, Durango.

${ }^{4}$ Becario de la Comisión de Operación y Fomento de Actividades Académicas del Instituto Politécnico Nacional.
}

González-E S, Rzedowski J. 1984. Algunos aspectos ecológicos y fitogeográficos de las especies de Cyperaceae en el Valle de México. II. Análisis fitogeográfico. Boletín de la Sociedad Botánica de México 46: 29-35. 
En esta contribución se pretende realizar un examen de afinidades geográficas de la flora de ciperáceas del Valle de México. Para algunos antecedentes se remite al lector al artículo previo (González y Rzedowski, 1983).

\section{COMPARACIÓN CON ALGUNAS OTRAS REGIONES DE la República MeXicana}

En la tabla 1 se confrontan los coeficientes de similitud determinados para los miembros de Cyperaceae del Valle de México con respecto a los de otras regiones de la República Mexicana, tanto a nivel de género como de especie. Las floras que se seleccionaron para comparación son las siguientes:

- Baja California (Wiggins, 1980), la cual puede considerarse como una flora completa.

- Parte norte de la altiplanicie mexicana, que comprende los estados de Coahuila, E de Chihuahua y parte de Durango y Zacatecas (Johnston, 1944), con una lista en la que ese autor considera que incluyó cerca de $75 \%$ de las plantas vasculares del área.

- Cuenca del río Mayo, en Sonora, y parte de Chihuahua (Gentry, 1942), con una lista florística incompleta.

- Nueva Galicia, que abarca los estados de Jalisco, Colima, Aguascalientes y parte de otros (Rzedowski y McVaugh, 1966), trabajo enfocado al análisis de la vegetación y sus relaciones con el medio, que incluye una lista florística parcial.

- Tabasco (P. Cowan, comunicación personal, 1980), consistente en una lista preliminar de las ciperáceas del estado.

- Península de Yucatán (O’Neill, 1940), en donde se estudian las ciperáceas de dicha área, con excepción de Rhynchospora.

- Los distritos de Soconusco y Mariscal, en el estado de Chiapas (Matuda, 1950), con una lista florística relativamente extensa.

Aunque en la tabla 1 se incluyen también los coeficientes a nivel de género, en este caso serán analizadas únicamente las relaciones a nivel de especie, ya que debido a la amplitud de distribución de los géneros de ciperáceas es posible que, al compararse áreas geográficas relativamente cercanas, sea poco significativo el uso del género como unidad de comparación.

TABLA 1. Coeficientes de similitud entre los miembros de la familia Cyperaceae del Valle de México y los de algunas otras regiones de la República Mexicana

\begin{tabular}{|c|c|c|c|c|c|c|c|}
\hline & 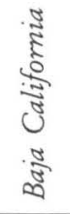 & 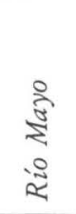 & $\frac{\sqrt[3]{3}}{0}$ & 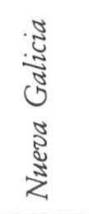 & 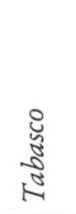 &  & 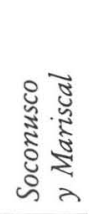 \\
\hline $\begin{array}{l}\text { Géneros } \\
\text { Especies y }\end{array}$ & 72.7 & 81.2 & 77.1 & 100.0 & 80.0 & 81.6 & 89.9 \\
\hline variedades & 28.8 & 35.0 & 40.6 & 53.4 & 16.2 & 20.4 & 23.9 \\
\hline
\end{tabular}


Los coeficientes de similitud (Cs) se obtuvieron usando las fórmulas propuestas por Preston (1962):

$$
\mathrm{T}=\left(\mathrm{A}^{1 / 0.27}+\mathrm{B}^{1 / 0.27}\right)^{0.27}
$$

donde A y B representan el número de taxa de cada lista florística analizada para comparación, y $\mathrm{T}$ es el número teórico total de taxa representados en ambas muestras si $\mathrm{A}$ y $\mathrm{B}$ fueran miembros de la misma flora. Para estas mismas condiciones teóricas, el número de taxa comunes entre $\mathrm{A}$ y $\mathrm{B}$ debe ser: $\mathrm{K}=\mathrm{A}+\mathrm{B}-\mathrm{T}$, y el coeficiente de similitud entre A y B es:

$$
\mathrm{CS}=\frac{100 \mathrm{~L}}{\mathrm{~K}} \%,
$$

donde $\mathrm{L}$ representa el número real de taxa comunes entre $\mathrm{A}$ y $\mathrm{B}$.

A nivel de especie se encontró una fuerte afinidad con Nueva Galicia (53.4\%), la cual puede explicarse en parte por la relativa cercanía geográfica entre las dos regiones comparadas, además de que en Nueva Galicia existen muchas áreas ecológicamente similares a las del Valle de México.

La península de Yucatán tiene uno de los índices de similitud más bajos a nivel de especie $(20.4 \%)$, lo que en parte se debe al aislamiento geográfico de esa región, y en parte a sus muy particulares características ecológicas. Pero, curiosamente, se encontró mayor afinidad con la península de Yucatán que con el estado de Tabasco. A pesar de que se conocen más especies de ciperáceas de Tabasco que del Valle de México, la mayor parte de éstas son típicas de regiones cálido húmedas, principalmente de sabanas, pastizales muy diferentes a los del Valle en composición y origen.

\section{COMPARACIÓN CON OTRAS REGIONES DEL MUNDO}

Usando el coeficiente propuesto por Preston, se analizó la relación entre las ciperáceas del Valle de México y las de algunas regiones situadas hacia el norte:

- Parte norte de Alaska (Wiggins y Thomas, 1962)

- Montañas Rocallosas en el estado de Colorado (Weber, 1972)

- Montañas Santa Cruz, California (Thomas, 1961)

- Estado de Arizona (Kearney y Peebles, 1951)

- Región del Trans-Pecos, Texas (Gould, 1962)

- Estado de Misisipí (Lowe, 1921)

- Polonia (Szafer, Kulczynski y Pawlowski, 1953)

Se compararon también regiones situadas al sur del Valle, incluyéndose en este grupo a la isla de Cuba, por poseer una flora con ligas muy fuertes con el sur.

- Panamá (Woodson, Schery et al., 1943)

- Cundinamarca, en el centro norte de Colombia (Mora-Osejo, 1966)

- Alrededores de Cumaná, Venezuela (Guevara-Z., 1974)

- Provincia de Buenos Aires, Argentina (Cabrera, 1968)

- Isla Barro Colorado, Panamá (Croat, 1978)

- Islas Galápagos (Wiggins y Porter, 1971)

- Cuba (León, 1946)

- Liberia (Nelmes y Baldwin, 1952) 
TABLA 2. Coeficientes de similitud entre los miembros de la familia Cyperaceae del Valle de México y los de otras regiones del mundo

(A) Al norte del Valle de México

\begin{tabular}{|c|c|c|c|c|c|c|c|}
\hline & 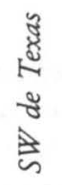 & 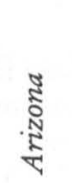 &  & 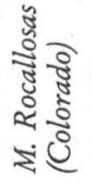 & 范 & $\begin{array}{l}\frac{z}{z} \\
\frac{3}{z} \\
z \\
z \\
z\end{array}$ & $\frac{\tilde{a}}{0}$ \\
\hline Géneros & 93.3 & 89.6 & 90.0 & 64.9 & 75.0 & 51.6 & 60.2 \\
\hline $\begin{array}{l}\text { Especies y } \\
\text { variedades }\end{array}$ & 33.2 & 38.8 & 18.6 & 12.6 & 10.9 & 0.0 & 6.5 \\
\hline
\end{tabular}

(B) Al sur del Valle, excepto Cuba

\begin{tabular}{|c|c|c|c|c|c|c|c|c|}
\hline &  & 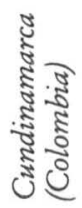 & 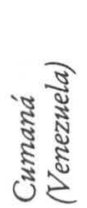 &  & 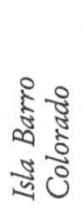 & 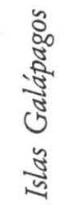 & $\frac{8}{3}$ &  \\
\hline Géneros & 100.0 & 99.9 & 77.1 & 100.0 & 74.6 & 81.2 & 100.0 & 69.9 \\
\hline $\begin{array}{l}\text { Especies y } \\
\text { variedades }\end{array}$ & 16.6 & 35.2 & 20.7 & 28.0 & 14.5 & 15.9 & 25.7 & 4.6 \\
\hline
\end{tabular}

En la tabla 2 (A) se compara la afinidad con varias regiones situadas del lado septentrional del área estudiada. Puede observarse que hay una disminución gradual del índice de similitud a medida que uno se aleja hacia el norte, excepto en los estados de Arizona y Misisipí: en Arizona la diferencia con el coeficiente esperado es casi insignificante; en cambio, en el estado de Misisipí la similitud con el Valle de México es relativamente baja, ya que a nivel de género supera apenas a las que hay con las Montañas Rocallosas, Alaska y Polonia, y a nivel de especie únicamente a las de Alaska y Polonia.

En la tabla 2 (B) se analiza la relación con regiones situadas al sur del Valle. Las primeras cuatro áreas están dentro del continente americano, y sus índices parecen indicar que a nivel de género hay una disminución gradual de la afinidad con el Valle a medida que se avanza hacia el sur, excepto en Buenos Aires, la más meridional de las regiones comparadas, donde el coeficiente se eleva a $100 \%$. A nivel de especie, la mayor relación es con el norte de Colombia, mientras que, paradójicamente, el índice más bajo se obtuvo de la relación con la región más cercana geográficamente (Panamá). Las siguientes tres regiones comparadas son áreas insulares. La relación más estrecha es con Cuba, tal vez debido a su relativa cercanía geográfica, mientras que la menor afinidad encontrada fue la de la isla Barro Colorado, a pesar de que las islas Galápagos están situadas más al sur. Por otro lado, también fue comparada una región del continente africano (Liberia), la cual muestra una afinidad relativamente fuerte con el Valle a nivel de género, pero casi nula a nivel de especie, reflejando la discontinuidad geográf ca y ecológica con el Valle de México.

En resumen, a nivel de género se encontró una ma 'or afinidad entre las ciperáceas del Valle y las de regiones de clima tropical. A nivel de especie, se registró una relación 
relativamente fuerte con Arizona y el SW de Texas, cuya flora puede ser considerada en buena parte como continuación de la del Altiplano mexicano, con la cual a su vez está muy ligada la del Valle de México. Esto reafirma la gran importancia del elemento autóctono entre las especies de ciperáceas del Valle. Se encontró también afinidad con las especies de una parte montañosa de Sudamérica (Cundinamarca), y con la provincia argentina de Buenos Aires, debidas seguramente a una mayor similitud de condiciones ecológicas entre esas áreas y el Valle de México.

\section{Distribución de las ciperáceas del Valle de México}

En la tabla 3 puede apreciarse que más de la mitad de los géneros del Valle se encuentran en regiones cálidas y templado cálidas del mundo; se trata de Abildgaardia, Bulbostylis, Cyperus, Fimbristylis y Rhynchospora. Los géneros cosmopolitas son Eleocharis y Scirpus, lo cual está relacionado con el hecho de que la mayor parte de sus especies son acuáticas o subacuáticas. Carex y Uncinia son de regiones templadas y frías, aunque Uncinia muestra una distribución marcadamente austral.

A nivel de especie, el elemento mejor representado es el endémico a México, con $28.9 \%$ del total de las especies del Valle, superando por un amplio margen a los que le siguen: especies americanas (14.5\%), especies distribuidas de México a Sudamérica (13.3\%), especies distribuidas del sur de Estados Unidos a Sudamérica (12.1\%), y elemento pantropical (9.6\%).

Considerando en forma más general los tipos de distribución, se observa que el elemento meridional (32.9\%) supera con mucho al boreal (12.2\%), y al pantropical y cosmopolita tomados juntos $(11.0 \%)$, así como al elemento americano $(14.5 \%)$. En esta disposición el elemento endémico queda reducido a segundo lugar en el orden de dominancia en el Valle (28.9\%), aunque sólo ligeramente superado por el elemento meridional.

TABLA 3. Distribución geográfica total de los géneros y especies de la familia Cyperaceae presentes en el Valle de México

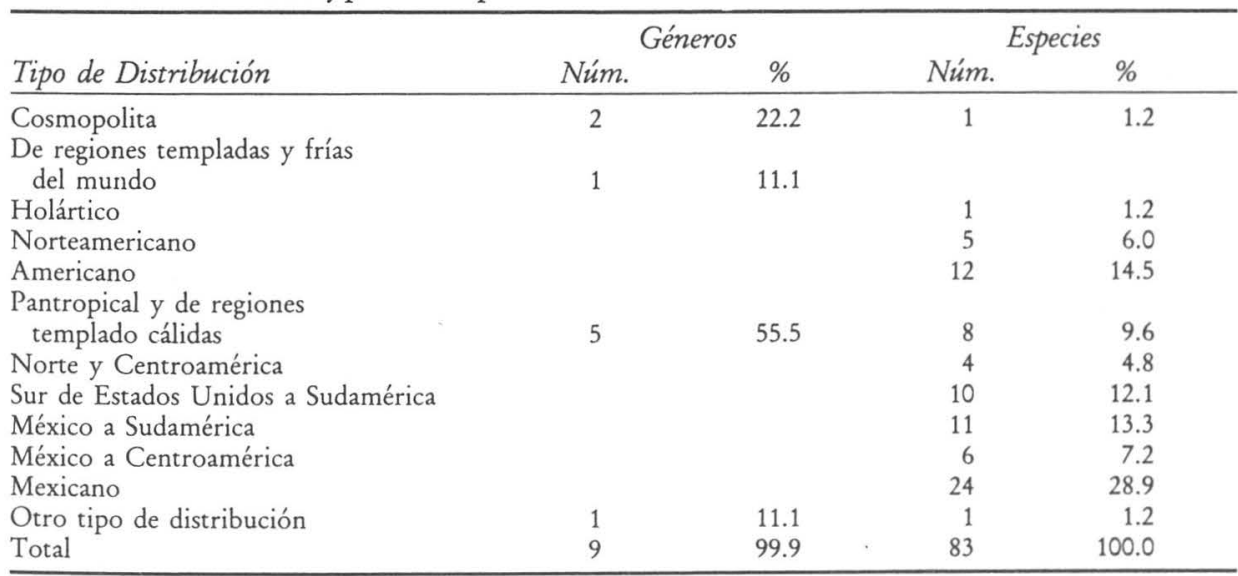


En cuanto a la relación entre la distribución general de las ciperáceas y su ubicación dentro del Valle de México, ocurre lo siguiente: los tipos de vegetación en donde se distribuyen principalmente las especies endémicas son, en orden decreciente: bosques de encino, pastizales, bosques de pino y matorrales xerófilos, aunque también hay unas pocas en bosques de Abies, en zacatonales subalpinos y en vegetación acuática y subacuática. Las únicas especies endémicas a México que se presentan en este último tipo de comunidad son Cyperus pycnostachyus, C. semiochraceus y Eleocharis densa.

El elemento meridional es de dos tipos: a] plantas de lugares bajos y templado cálidos, localizadas en pastizales, matorrales xerófilos y vegetación acuática y subacuática, $\mathrm{y}$ b] plantas de lugares altos con clima templado o frío, localizadas en bosques de encino, bosques de oyamel y bosques mesófilos de montaña; entre estas últimas destacan algunas especies distribuidas en forma aparentemente disyunta en las montañas de México y Sudamérica, como es el caso de Carex brachycalama y Uncinia phleoides.

Las afinidades con el norte se presentan principalmente en bosques de pino y zacatonales alpinos y subalpinos, y en muy pequeña escala en bosques de Abies.

Las especies de amplia distribución son mayormente acuáticas o subacuáticas, aunque algunas se conocen de pastizales, bosques de encino, matorrales xerófilos y de comunidades de plantas arvenses y ruderales.

La diversificación de la familia a nivel de género es mayor cerca del ecuador, aunque en algunas regiones a altas latitudes, por ejemplo Polonia, es también excepcionalmente grande. A nivel de especie el grupo está también muy bien representado en los trópicos, debido a la venturosa evolución de varios géneros, principalmente Cyperus, pero a medida que se avanza hacia los polos, la mayor parte de las especies corresponden al género Carex.

De acuerdo con Raven y Axelrod (1974), la familia evolucionó inicialmente en regiones tropicales y subtropicales, donde existe la mayor diversidad de linajes primitivos, emigrando de ahí hacia el norte y el sur, y el género más grande y especializado (Carex) es el dominante en regiones frías. Los resultados del presente análisis coinciden con lo anterior.

\section{LITERATURA CITADA}

Cabrera, A.L. 1968. Flora de la Provincia de Buenos Aires. I. Colección Científica del inta, Buenos Aires. Croat, T.B. 1978. Flora of Barro Colorado Island. Stanford Univ. Press. Stanford, Calif. 943 p.

Gentry, H.S. 1942. Rio Mayo plants. Carnegie Inst. Washington. Publ. 527. Washington, D.C. 328 p.

GONZÁLEZ, S., y J. RzEDOWSKI. 1983. Algunos aspectos ecológicos y fitogeográficos de las especies de Cyperaceae en el Valle de México. I. Afinidades ecológicas. Bol. Soc. Bot. México 45:39-47.

Gould, F.W. 1962. Texas plants. A checklist and ecological summary. Agric. Mech. Coll. Texas. Texas Agric. Exp. Stn. 112 p.

Guevara-Z., M.C. 1974. Estudio taxonómico de la familia Cyperaceae de Cumaná y sus alrededores. Tesis. Universidad de Oriente. Cumaná, Venezuela, 125 p.

Johnston, I.M. 1944. Plants of Coahuila, eastern Chihuahua and adjoining Zacatecas and Durango. III. J. Arnold Arbor. 25:43-83, 133-183, 431-453.

Kearney, T.H., y R.H. Peebles. 1951. Arizona Flora. Univ. Calif. Press, Berkeley. 1032 p.

León, Hno. 1946. Flora de Cuba. I. Contribuciones ocasionales del Museo de Historia Natural del Colegio La Salle 8:183-236.

Lowe, E.N. 1921. Plants of Mississippi. A list of flowering plants and ferns. Mississippi Geol. Surv. 292 p. Matuda, E. 1950. A contribution to our knowledge of the wild and cultivated flora of Chiapas. I. Amer. Midland Nat. 44(3):513-616. 
Mora-Osejo, E. 1966. Catálogo ilustrado de las plantas de Cundinamarca. Vol. I. Cyperaceae. Inst. de Ciencias Naturales. Univ. Nacional. Bogotá: 95-133.

Nelmes, E. y J. Baldwin. 1952. Cyperaceae in Liberia. Amer. J. Bot. 39(6):368-393.

O'NeILL, H.T. 1940. The sedges of the Yucatan Peninsula. Carnegie Inst. Washington. Publ. 522. Washington, D.C.: $247-322$.

PRESTON, F.W. 1962. The canonical distribution of commonness and rarity. Ecology 43:185-215, 410-431.

Raven, P. y D. AXELrod. 1974. Angiosperm biogeography and past continental movements. Ann. Missouri Bot. Gard. 61(3):539-673.

Rzedowski, J. y R. McVaugh. 1966. La Vegetación de Nueva Galicia. Contr. Univ. Mich. Herb. 9:1-123.

Szafer, W., S. Kulczynski y B. Pawlowski. 1953. Rosliny Polskie. Państwowe Wydawnictwo Naukowe. Warszawa. 1020 p.

Thomas, J.H. 1961. Flora of the Santa Cruz mountains of California. Stanford University Press, Stanford, Calif. $434 \mathrm{p}$.

WEBER, W.A. 1972. Rocky Mountain flora. Colorado Associated University Press. Boulder, Colorado. 438 p.

Wiggins, I.L. 1980. Flora of Baja California. Stanford University Press. Stanford, Calif. 1025 p. y D.M. PorTer, 1971. Flora of the Galapagos Islands. Stanford University Press, Stanford, Calif. 998 p. y J.H. Thomas, 1962. A flora of the Alaskan arctic slopes. University of Toronto Press, Toronto. 425 p.

Woodson, R.E., R.W. Schery y colaboradores. 1943. Flora of Panama II. (2). Ann. Missouri Bot. Gard. $30(3): 281-325$. 